\title{
Compliance with periodic surveillance for Von-Hippel-Lindau disease
}

\author{
Chantal R. M. Lammens, $P h D^{I}$, Neil K. Aaronson, PhD ${ }^{l}$, Frederik J. Hes, MD, $P h D^{2}$, \\ Thera P. Links, MD, PhD ${ }^{3}$, Bernard A. Zonnenberg, MD, PhD ${ }^{4}$, Jacques W. M. Lenders, MD, PhD ${ }^{5,6}$, \\ Danielle Majoor-Krakauer, MD, $P h D^{7}$, Theo A. M. Van Os, MD ${ }^{8}$, Encarna B. Gomez-Garcia, MD, PhD', \\ Wouter de Herder, MD, PhD ${ }^{10}$, Rob B. van der Luijt, PhD ${ }^{11}$, Ans M. W. van den Ouweland, PhD ${ }^{7}$, \\ Liselot P. Van Hest, $M D^{12}$, Senno Verhoef, $M D, P h D^{13}$, and Eveline M. A. Bleiker, PhD ${ }^{1,13}$
}

\begin{abstract}
Purpose: To assess compliance with a periodic surveillance regimen for Von Hippel-Lindau disease. Methods: In this nationwide study, Von Hippel-Lindau disease mutation carriers and those at 50\% risk were invited to complete a questionnaire assessing (compliance with) advice given for periodic surveillance. Medical record data on compliance with recommended radiologic surveillance examinations were also collected. Results: Of the 84 (77\%) participants, 78 indicated having received advice to undergo periodic surveillance. Of these, 71 reported being fully compliant with that advice. In $64 \%$ of the cases, this advice was only partially consistent with published guidelines. Based on medical record data, between one quarter and one third of individuals did not undergo surveillance as recommended in the guidelines for central nervous system lesions and one half for visceral lesions. Screening delay for central nervous system lesions was significantly higher in one hospital and in those cases where "the advice given" deviated from the guidelines. Conclusions: The majority of those with or at risk of Von Hippel-Lindau disease reported having received and being fully compliant with screening advice. However, in many cases, the advice given was only partially consistent with published guidelines, and screening delays were observed. Efforts should be undertaken to stimulate guideline-based surveillance advice and to minimize screening delay. Genet Med 2011:13(6):519-527.
\end{abstract}

Key Words: Von Hippel-Lindau disease (VHL), periodic surveillance, guidelines, compliance, psychosocial impact

From the ${ }^{1}$ Department of Psychosocial Research and Epidemiology, The Netherlands Cancer Institute-Antoni van Leeuwenhoek hospital (NKI-AVL), Amsterdam; ${ }^{2}$ Department of Clinical Genetics, Leiden University Medical Centre, Leiden; ${ }^{3}$ Department of Internal Medicine, University Medical Centre Groningen, Groningen; ${ }^{4}$ Department of Internal Medicine, University Medical Centre Utrecht, Utrecht; ${ }^{5}$ Department of Internal Medicine, University Nijmegen Medical Centre, Nijmegen, The Netherlands; ${ }^{6}$ Department of Medicine III, Carl Gustav Carus University Medical Center, Dresden, Germany; ${ }^{7}$ Department of Clinical Genetics, Erasmus Medical Centre, Rotterdam; ${ }^{8}$ Department of Clinical Genetics, Amsterdam Medical Centre, Amsterdam; ${ }^{9}$ Department of Clinical Genetics, Academic Hospital Maastricht, Maastricht; ${ }^{10}$ Department of Internal Medicine, Erasmus Medical Centre, Rotterdam; ${ }^{11}$ Department of Clinical Genetics, University Medical Centre Utrecht, Utrecht; ${ }^{12}$ Department of Clinical Genetics, VU medical centre, Amsterdam; and ${ }^{13}$ The Netherlands Cancer Institute-Antoni van Leeuwenhoek hospital (NKI-AVL), Family Cancer Clinic, Amsterdam, The Netherlands.

Eveline M. A. Bleiker, PhD, Department of Psychosocial Research and Epidemiology, The Netherlands Cancer Institute-Antoni van Leeuwenhoek hospital (NKI-AVL), Plesmanlaan 121, Amsterdam 1066 CX, The Netherlands, E-mail: e.bleiker@nki.nl.

Disclosure: The authors declare no conflict of interest.

Submitted for publication August 6, 2010.

Accepted for publication November 30, 2010.

Published online ahead of print March 16, 2011

DOI: 10.1097/GIM.0b013e3182091a1d $\bigvee_{n=1}^{\circ}$ on Hippel-Lindau disease (VHL) is an autosomal, dominantly inherited tumor susceptibility syndrome that is characterized by an increased risk of developing multiple benign tumors and malignant neoplasms. These tumors are often multicentric or bilateral. ${ }^{1}$ The most prevalent tumors are hemangioblastomas of the retina, cerebellum, or myelum; pheochromocytomas; renal cysts; renal clear cell carcinomas; and cysts and endocrine tumors of the pancreas. ${ }^{2,3}$ Renal clear cell carcinoma metastasis and neurologic damage due to central nervous system (CNS) hemangioblastomas are the most common cause of death. ${ }^{3-7}$ The cause of VHL is a germline mutation in the $V H L$ gene. ${ }^{8}$ At least $90 \%$ of the carriers of a $V H L$ mutation exhibit clinical manifestations of VHL by the age of 60 years. Without treatment, the median expected survival of patients with VHL has been estimated to be 49 years of age. ${ }^{3}$ The age of onset and expression of the disease vary widely (e.g., age 1-67 years for retinal hemangioblastomas; age 5-70 years for pancreatic cancers or cysts; and age 16-67 years for renal cell carcinoma $[\mathrm{RCC}]$ or cysts). ${ }^{2}$

Currently, there are no preventive strategies available to avoid the occurrence of tumors, and no prophylactic surgery is available. However, early diagnosis and treatment, such as laser treatment for retinal hemangioblastomas at an asymptomatic stage, may affect prognosis positively. Therefore, high-risk individuals are advised to undergo periodic, multidisciplinary surveillance according to published, national guidelines. ${ }^{9}$ These national guidelines are largely in line with international guidelines. ${ }^{2,9,10}$

In the Netherlands, surveillance begins at age 5 years with an annual ophthalmologic examination (Table 1). ${ }^{9}$ Although no large empirical studies on the effectiveness of regular surveillance for VHL are available, it is generally believed that the introduction of periodic surveillance and the improvement in surgical techniques have contributed to the substantial decrease in morbidity and mortality in this population..$^{2,11-14}$ Research is ongoing to determine the most optimal intervals between screenings. ${ }^{12}$ Because of the beneficial effects of regular surveillance for VHL, it is important that individuals diagnosed with or at high risk of VHL receive and adhere to surveillance advice that is consistent with the VHL surveillance guidelines.

Only one recent study has reported on periodic surveillance for VHL. ${ }^{15}$ Of the 36 identified carriers, all underwent initial surveillance for VHL manifestations after receiving their genetic test result. However, after a 5-year follow-up, only $39 \%$ were adherent with the advised periodic surveillance program. The symptomatic carriers were more likely to be adherent than the asymptomatic carriers. ${ }^{15}$ It should be noted that, in this study, nonadherence with the recommended surveillance protocol was not clearly defined. Furthermore, 10 of the 36 carriers in the study were younger than 18 years. It is most likely that noncompliance of the minors depends on the compliance be- 


\begin{tabular}{lcc}
\hline Table 1 Dutch VHL guidelines for regular surveillance \\
\hline Investigation & Age & Frequency \\
\hline Ophthalmologic examination & From $5 \mathrm{yr}$ & Annually \\
Anamnesis & From $10 \mathrm{yr}$ & Annually \\
Physical examination, blood pressure & From $10 \mathrm{yr}$ & Annually \\
Biochemical blood tests & From $10 \mathrm{yr}$ & Annually \\
24-hr urine test & From $10 \mathrm{yr}$ & Annually \\
Upper abdominal ultrasound & From $10 \mathrm{yr}$ & Annually \\
MRI cerebellum and myelum & From $15 \mathrm{yr}$ & Biannually \\
MRI upper abdomen & When indicated & \\
MRI inner ear & When indicated & \\
Audiogram & When indicated & \\
Neurologic examination & When indicated & \\
\hline
\end{tabular}

havior of the parents. Additionally, psychosocial questionnaires were only administered to 17 adult carriers.

This study was undertaken to assess compliance with periodic surveillance for VHL. The main aims of this study were to investigate (1) the surveillance advice given to high-risk family members; (2) the concordance between the advice given and the national VHL surveillance guidelines; (3) the degree of compliance; and (4) variables associated significantly with noncompliance.

\section{MATERIALS AND METHODS}

\section{Study sample and procedures}

As part of a larger, ongoing nationwide cross-sectional study performed in collaboration with all nine family cancer clinics in the Netherlands and the DNA-laboratories of the University Medical Center Utrecht and the Erasmus Medical Center in Rotterdam, 48 families with a known pathogenic VHL germline mutation were identified. ${ }^{16}$ Five of these families were excluded from this study because all the family members registered at the family cancer clinics were deceased, had emigrated, or were younger than 16 years. In total, 109 family members (from the remaining 43 families) with a clinical and/or molecular genetic diagnosis of VHL or at $50 \%$ risk of VHL were invited to participate in the study. Individuals were recruited between August 2006 and February 2008.

Those eligible received a letter of invitation from their clinical geneticist, along with an information leaflet about the study, a consent form, a questionnaire, and a prepaid return envelope. Eligible individuals who were not registered at a clinical genetics center were invited by a registered family member. Adult carriers were requested to invite their children aged $16-18$ years to participate in the study.

\section{Measures}

\section{Sociodemographic and clinical variables}

Participants' age, gender, marital status, level of education, offspring, hospital, DNA status (carrier or 50\% at risk), and disease status (affected/unaffected) were obtained from the selfreport questionnaire and the medical records.

\section{Advice for periodic surveillance}

A self-report questionnaire was used to assess advice given for periodic surveillance (study part I). Respondents indicated what type of advice, if any, for periodic surveillance they had received on a given list with possible surveillance methods and interval options (based on the national guidelines). This allowed us to compare self-reported advice with the surveillance program as recommended in the national guidelines (Table 1).

\section{Compliance with periodic surveillance}

Compliance with periodic surveillance was assessed in two ways.

Study part I. First, respondents were asked to report the extent to which they had adhered to the (self-reported) advice that they had received for regular surveillance. Self-reported compliers were those high-risk family members who indicated that they had followed the surveillance advice "as advised" or "more frequently than advised." Self-reported noncompliers were those individuals who reported not having followed the surveillance advice that they indicated they had received or did so less frequently than advised. Additionally, reasons for noncompliance were asked, including "the discomfort of the surveillance method(s)," because it was "too time consuming," because of "difficulties in planning the examinations," because of "absence of symptoms," and/or because they believed that the probability of something being detected was very small.

Study part II. Second, medical records data were used to determine objective compliance (surveillance performed in accordance with the guidelines) with the radiologic surveillance examinations, including magnetic resonance imaging (MRI) of the CNS and ultrasound of the upper abdomen. We focused on these two screening methods because manifestations detected by radiologic examinations (e.g., CNS lesions and RCC) have the most severe consequences, and these data could most reliably be collected from the medical records. Specifically, data on the number of radiologic surveillance procedures and intervals between two concurrent surveillance sessions were retrieved from the medical records for the period January 2004 to December 2008.

Based on the national guidelines for periodic surveillance, decision rules for classifying a minimal level of objective compliance were developed and formulated as follows: (1) for the MRI of the cerebellum and myelum, a minimum of two examinations per 5-year period are required, and an interval of $>27$ months between two consecutive examinations is considered unacceptable and (2) for compliance with the imaging surveillance methods of the upper abdomen (ultrasound or other imaging techniques), a minimum of four examinations is required, and an interval between two consecutive examinations of $>15$ months is considered unacceptable. Based on these criteria, respondents were classified either as compliant (surveillance as advised in the national surveillance guidelines) or undercompliant (too few examinations or too lengthy a delay between examinations).

\section{Psychosocial data}

To investigate which variables were associated with "objective surveillance compliance," a series of questions was posed based on the central elements of two social cognition models of preventive health behavior, the Health Belief Model, ${ }^{17}$ and the Protection Motivation Theory. ${ }^{18}$

Perceived risk. Respondents were asked to report their perceived risk of developing a(nother) tumor compared with that of 
the "average person in the Dutch population" (item adapted from Lerman et al.). ${ }^{19}$ Response categories ranged on a fivepoint scale, from "lower" to "much higher."

Perceived benefits of and barriers to periodic surveillance. Perceived benefits and barriers were assessed with an 11-item scale adapted from previous work of Champion, ${ }^{20} \mathrm{Kash}$ et al., ${ }^{21}$ and Madalinska et al. ${ }^{22}$ This included five "pro" statements and six "con" statements about periodic surveillance. Response categories ranged on a five-point scale from "strongly disagree" to "strongly agree." Sum scores for the pro and con subscales ranged from 5 to 25 , and 6 to 30 , respectively. The reliability (Cronbach's coefficient alpha) of the pro and con subscales in our study group was 0.83 and 0.53 , respectively.

VHL-specific distress. VHL-specific distress was measured with the Dutch version of the "intrusion" subscale of the Impact of Events Scale. ${ }^{23}$ This seven-item questionnaire measures intrusive thoughts and feelings about VHL during the past 7 days. The Impact of Event Scale-intrusion total score ranges between 0 and 35. A score of 9 or higher is considered to be clinically relevant, ${ }^{24}$ and additional psychosocial help may be indicated. Cronbach's alpha for the intrusion scale in this study was 0.91 .

VHL-related worries. VHL-related worries were assessed with an eight-item questionnaire adapted from the Cancer Worry Scale. ${ }^{25,26}$ Scores range from 8 to 32, with higher scores indicating more frequent worries about cancer. Cronbach's alpha in this study was 0.89 .

\section{Data analysis}

Descriptive statistics were generated to characterize the study sample in terms of sociodemographic and clinical background variables.

\section{Study part I}

Descriptive statistics were used to document the recommended surveillance methods (self-reported advice) and to investigate the extent to which the self-reported advice on regular surveillance was in accordance with the national surveillance guidelines, ${ }^{9}$ to assess self-reported compliance with surveillance, to describe reasons for self-reported noncompliance, and to identify the perceived barriers and benefits of regular surveillance for VHL. In assessing concordance, the medical consultation (including an annual anamnesis and physical examination) and those examinations advised and performed "on indication only" were excluded from the analysis, as these were difficult to measure reliably.

\section{Study part II}

Descriptive statistics were used to summarize the objectively determined compliance with the radiologic surveillance methods. In addition, univariate analyses (Student's $t$-test, Fisher's exact test, and $\chi^{2}$ test) were used to determine which sociodemographic, clinical, and psychological variables were related significantly to objective undercompliance (screening delay). The variable "hospital" was coded into four categories; one category each for the three hospitals with the largest number of participants, and the remaining category for all other hospitals. It was not possible to control for potential clustering effects (i.e., multiple family members from the same family) because multiple families had only one member who participated in the study. All analyses were conducted using the Statistical Package for the Social Sciences (version 17.0).

\section{RESULTS}

\section{Response and sample characteristics}

In total, $84(77 \%)$ of the 109 invited high-risk individuals stemming from 36 VHL families completed the questionnaire, including 68 carriers and 16 family members at 50\% risk. There were no statistically significant differences between respondents and nonrespondents in terms of age, gender, or actual risk status (carrier vs. $0 \%$ at risk).

Characteristics of the study participants are presented in Table 2. The respondents' ranged in age between 16 and 65 years (mean $=38.1$ years; $\mathrm{SD}=13.7$ years). Gender was equally distributed. The majority of the participants were carriers, of whom most had one or more VHL manifestations.

\section{Study part I}

\section{Self-reported advice for periodic surveillance}

Of the 84 participating high-risk family members, six (7\%) stated that they had not received an advice to undergo periodic surveillance for VHL. All six family members had a 50\% risk of having inherited the VHL gene mutation and had not undergone genetic testing. Four stemmed from the same family. All but one of these individuals belonged to families registered at the family cancer clinic of one particular hospital.

\section{Concordance between self-reported advice and the guidelines}

Overall. Of the 78 respondents who reported having received advice on regular surveillance, $28(36 \%)$ received advice that was consistent with the national VHL guidelines (Fig. 1). Of those $(n=50)$ for whom the advice received was inconsistent

Table 2 Characteristics of the study sample $(N=84)$

\begin{tabular}{lc}
\hline & $N(\%)$ \\
\hline Age (mean $\pm \mathrm{SD})$ & $38.1 \pm 13.7$
\end{tabular}

Actual risk

(a)symptomatic carriers

$50 \%$ at risk

VHL manifestation(s)

Yes

No

Gender

Male

Female

Marital status

Married/living together

Single

Children (yes)

Educational level

Low

Moderate

High 


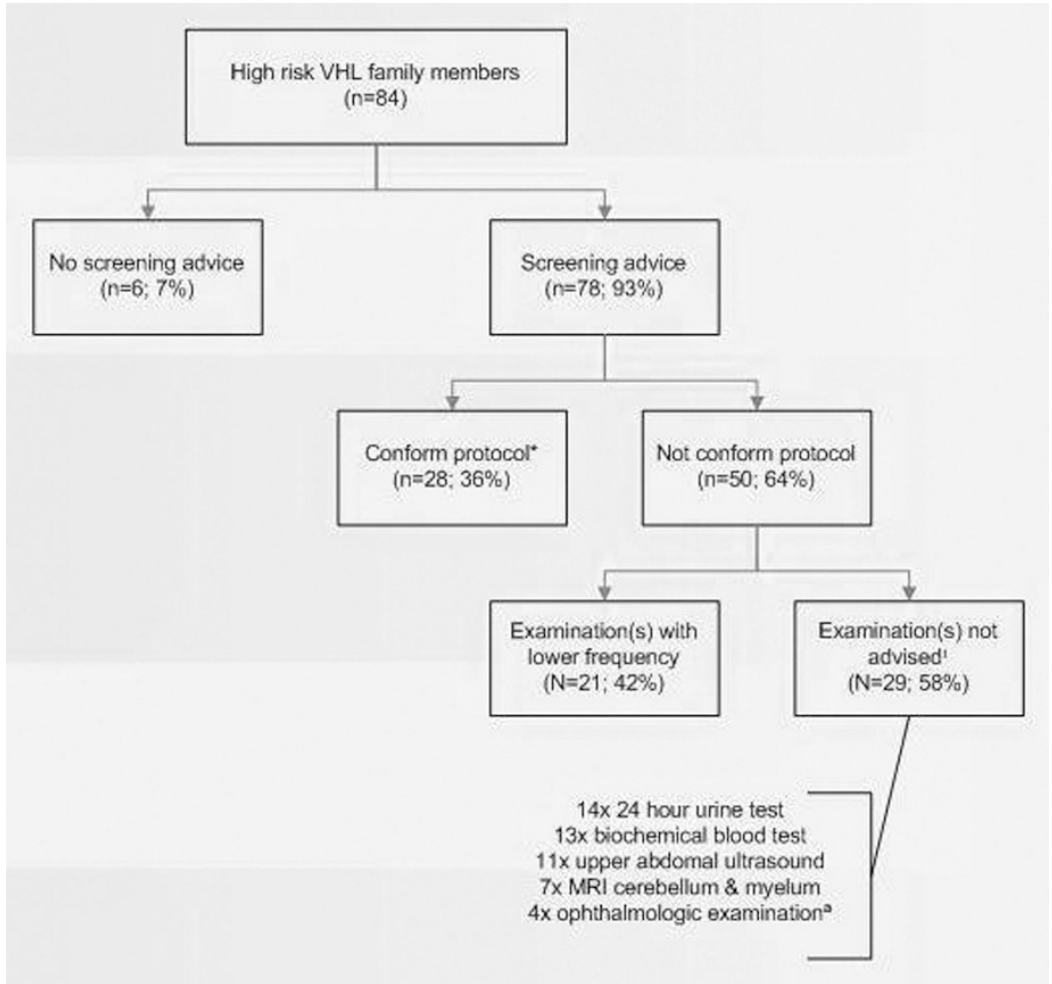

Fig. 1. Regular surveillance: advice and extent to which advice for regular surveillance conforms to the national surveillance guidelines. *Conform protocol: all examinations conform to the Dutch national guidelines (Table 1), excluding medical consultation (annual anamnesis and physical examination) and those only if there is an indication. The median number of examinations that were not advised is 1 (55\%; range: $1-4)$. ${ }^{a}$ One high-risk individual underwent a bilateral enucleation of the eyes; therefore, no ophthalmologic examination was advised.

Table 3 Regular surveillance: advice and extent to which advice for regular surveillance conforms to the national surveillance guidelines per surveillance method $(n=78)$

\begin{tabular}{|c|c|c|c|c|}
\hline & $\begin{array}{l}\text { Examination } \\
\text { advised } \\
(n=78) \\
\quad N(\%)\end{array}$ & $\begin{array}{l}\text { Advice } \\
\text { conform } \\
\text { guidelines, } \\
\quad N(\%)\end{array}$ & $\begin{array}{l}\text { Advice not } \\
\text { conform } \\
\text { guidelines, } \\
\quad N(\%)\end{array}$ & $\begin{array}{c}\text { Range } \\
\text { "too low } \\
\text { frequency" } a\end{array}$ \\
\hline $\begin{array}{l}\text { Medical } \\
\quad \text { consultation }^{b}\end{array}$ & $65(83)$ & $59(91)$ & $6(9)$ & $1.5-3 \mathrm{yr}$ \\
\hline $\begin{array}{l}\text { Ophthalmologic } \\
\text { examination }\end{array}$ & $74(95)^{c}$ & $58(78)$ & $16(22)$ & $1.5-2 \mathrm{yr}$ \\
\hline $\begin{array}{l}\text { Biochemical } \\
\text { blood tests }\end{array}$ & $65(83)$ & $55(85)$ & $10(15)$ & $1.5-5 \mathrm{yr}$ \\
\hline 24-hr urine test & $64(82)$ & $53(83)$ & $11(17)$ & $1.5-5 \mathrm{yr}$ \\
\hline $\begin{array}{l}\text { Upper abdominal } \\
\text { ultrasound }^{d}\end{array}$ & $67(86)$ & $53(79)$ & $14(21)$ & $1.5-2 \mathrm{yr}$ \\
\hline $\begin{array}{l}\text { MRI cerebellum } \\
\text { and myelum }\end{array}$ & $71(91)$ & $66(93)$ & $5(7)$ & $\begin{array}{l}5 \text { yr or if } \\
\text { symptomatic }\end{array}$ \\
\hline \multicolumn{5}{|c|}{$\begin{array}{l}{ }^{a} \text { The range of deviations from the recommended frequency in the guidelines. } \\
{ }^{b} \text { Includes annual anamnesis and physical examination. } \\
{ }^{c} \text { One high-risk individual underwent a bilateral enucleation of the eyes, and } \\
\text { therefore, no ophthalmologic examination was advised. } \\
{ }^{d} \text { Or other radiological examination of the upper abdomen. }\end{array}$} \\
\hline
\end{tabular}

with the guidelines, $29(58 \%)$ had receive advice to undergo some but not all the recommended screenings. The remaining 21 respondents $(42 \%)$ reported having been advised to undergo all the screening procedures found in the guidelines, but the recommended frequency of screening deviated from the guidelines for one or more of the procedures. The range of deviations from the recommended frequency in the guidelines per screening procedure is presented in Table 3 (Fig. 1).

Per surveillance method. Table 3 displays the number of individuals for whom the self-reported screening advice was consistent with national guidelines, per screening method. Sixtyseven individuals (86\%) indicated that they had received advice to undergo periodic radiologic examinations of the upper abdomen; in 53 of these cases $(79 \%)$, the frequency of this advice was consistent with the national guidelines. Additionally, 71 individuals $(91 \%)$ received advice to undergo an MRI of the cerebellum and myelum; in 66 of these cases (93\%), this advice was consistent with the guidelines. Of the remaining five individuals (7\%), four reported having been advised to undergo an MRI of the cerebellum and myelum only if they had symptoms, and one individual reporting having been advised to undergo an MRI only once every 5 years.

\section{Compliance with self-reported advice}

Of the 78 individuals who indicated having been advised about periodic surveillance, 71 (91\%) stated that they had been fully compliant with the advice given (i.e., that they had undergone all surveillance methods as frequently or more frequently 
than advised). Of the seven individuals ( $9 \%$ ) who reported not having been compliant, five were symptomatic carriers and two were at $50 \%$ risk

\section{Reasons for noncompliance with self-reported advice}

The two "at-risk" noncompliers indicated that they did not undergo regular screening because they did not have any health complaints and, thus, considered the screening to be unnecessary. Additionally, one of these individuals reported being fearful of the MRI scan (i.e., claustrophobia) and of the possibility that a tumor would be detected. Reasons for noncompliance given by the five symptomatic carriers included the personal need for rest between periods of illness and/or hospitalization $(n=2)$, fear of the MRI scan $(n=2)$, the perceived burden of the surveillance $(n=2)$, and fear that a tumor would be detected $(n=1)$.

\section{Perceived benefits and barriers of periodic surveillance}

The most prevalent perceived benefits of surveillance were early detection of a VHL-related tumor (97\%) and gaining a sense of security $(73 \%)$. The most important perceived barriers to surveillance were that it would cause unnecessary worry $(47 \%)$ and that it was impractical or inconvenient (e.g., in relationship to work and childcare due to planning of frequent hospital visits; 32\%). Overall, self-reported compliers reported significantly more benefits from surveillance than noncompliers $(P<0.001)$. No significant differences were found regarding the perceived barriers of surveillance (Table 4).

\section{Study part II}

\section{Objective compliance with radiological examinations}

Objective compliance data (i.e., surveillance performed in accordance with the guidelines) were obtained from the medical records. Of those individuals who indicated not having received advice for periodic surveillance $(n=6)$, indeed no radiologic data were found in the medical files of the participating academic medical centers. Medical record data on periodic radiologic surveillance were available for 67 of the 78 individuals (86\%) who reported having received surveillance advice for CNS lesions. Data on surveillance of the upper abdomen were available for 64 individuals $(82 \%)$.

Based on the decision rules for determining objective compliance with the radiologic examinations, as described in the "Materials and Methods," 24\% $(n=16)$ and 34\% $(n=23)$, respectively, did not undergo surveillance in accordance with the guidelines for lesions in the cerebellum or myelum (Table $5)$. Of the 16 who were undercompliant with the radiologic examinations of the cerebellum, 12 (75\%) underwent fewer than two MRIs in the previous 5-year period. Additionally, for four individuals $(25 \%)$, the interval between two consecutive MRI scans was too long ( $>27$ months). Of the 23 undercompliers with the radiologic examinations of the myelum, $21(91 \%)$ underwent fewer than two MRIs during the previous 5-year period, and for the remaining two (9\%) individuals, the interval between two consecutive surveillance data were too long. Among the delayers, the mean deviating time interval outside the recommended range between two consecutive MRIs of the

Table 4 Perceived benefits of and barriers to surveillance for VHL $(n=78)$

Total $(n=78)$

$97 \%$

Reduces the chance of a tumor being detected in an advanced stage

Reduces my fear of developing a tumor

Provides me with a feeling of control

Gives me a sense of security

Is a good way to detect a tumor early

Total score benefits (mean $\pm \mathrm{SD}$ )

Barriers to surveillance

Can have a negative effect on my home mortgage and/or life and health insurance ${ }^{b}$

Can cause unnecessary worry

Causes inconvenience in my life

Screening is not important, if I am diagnosed with a tumor it will be too late

Is painful

Is uncomfortable/embarrassing
$36 \%$

$35 \%$

$73 \%$

$97 \%$

$18.6(3.2)$

$27 \%$

$47 \%$

$32 \%$

$3 \%$

$6 \%$

$24 \%$

$14.1(3.6)$
Adherent $(n=71)$

Not (fully) adherent $(n=7)$

Total score barriers (mean $\pm \mathrm{SD}$ )

$100 \%^{a}$

$39 \%^{a}$

$37 \%$

$80 \%^{a}$

$99 \%$

$19.1 \pm 3.0^{a}$

$73 \%$

$71 \%^{a}$

$46 \%$

$57 \%$

$31 \%$

$43 \%$

$1 \%$

$14 \%$

$14 \%$

$6 \%$

$22 \%$

$43 \%$

${ }^{a}$ Significant difference $P \leq 0.05$.

${ }^{b}$ One case missing in analysis. 
Table 5 Objective screening compliance with radiological examinations in high-risk VHL family members ${ }^{a}$

\begin{tabular}{|c|c|c|c|c|}
\hline & \multirow[b]{2}{*}{$\begin{array}{l}\text { Compliant, } \\
\quad N(\%)\end{array}$} & \multirow[b]{2}{*}{$\begin{array}{l}\text { Not compliant, } \\
\qquad N(\%)\end{array}$} & \multicolumn{2}{|c|}{ Reason for noncompliance } \\
\hline & & & $\begin{array}{l}\text { Limited number of } \\
\text { examinations, }{ }^{b} N(\%)\end{array}$ & $\begin{array}{c}\text { Interval between two examinations } \\
\text { is too long, }{ }^{c} N(\%)\end{array}$ \\
\hline MRI cerebellum ${ }^{d}(n=67)$ & $51(76)$ & $16(24)$ & $12(75)$ & $4(25)$ \\
\hline MRI myelum ${ }^{e}(n=67)$ & $44(66)$ & $23(34)$ & $21(91)$ & $2(9)$ \\
\hline Ultrasound upper abdomen ${ }^{f g}(N=64)$ & $33(52)$ & $31(48)$ & $16(52)$ & $15(48)$ \\
\hline \multicolumn{5}{|c|}{ 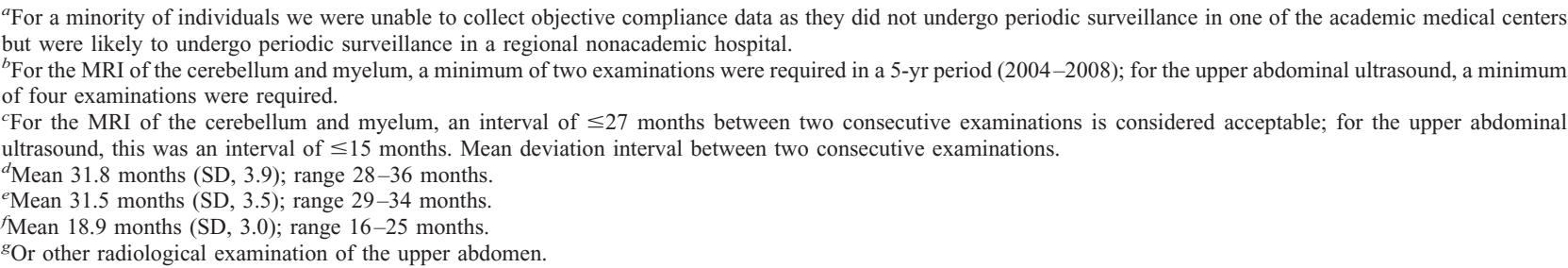 } \\
\hline
\end{tabular}

cerebellum or myelum was 31.8 (SD: 3.9; range: 28-36) and 31.5 (SD: 3.5; range: 29-34) months, respectively.

With respect to the radiologic examinations for visceral lesions, $48 \%(n=31)$ did not undergo surveillance as recommended. Of these 31 individuals, 16 (52\%) underwent too few examinations, and for 15 individuals (48\%), the intervals between one or more consecutive surveillance data were too long ( $>15$ months). Among those who delayed, the mean deviating time interval outside the recommended range between two consecutive radiologic examinations of the upper abdomen was 18.9 months (SD: 3.0; range: 16-25).

\section{Variables associated with undercompliance}

Undercompliance (screening delay) with radiologic examinations for CNS lesions (i.e., too few examinations or too long an interval between examinations) was associated significantly with having received (based on self-report) advice for periodic surveillance that deviated from the guidelines $(P<0.01)$. Additionally, undercompliance was significantly higher in one hospital $(P<0.01)$. Compliance with surveillance for CNS lesions was not associated significantly with the actual risk status of the respondents, perceived risk, benefits or barriers of surveillance, or levels of psychosocial distress or worries (Table 6).

None of the variables investigated were found to be associated significantly with compliance with radiologic examinations of the upper abdomen to detect visceral lesions. However, unaffected individuals $\left(P_{\text {trend }}=0.08\right)$ and those with lower levels of distress $\left(P_{\text {trend }}=0.08\right)$ and worries $\left(P_{\text {trend }}=0.07\right)$ tended to be less compliant (Table 6).

\section{DISCUSSION}

For individuals diagnosed with or at high risk of VHL, periodic surveillance is important to ensure early diagnosis and treatment. To our knowledge, this study is the first to investigate the advice given for periodic surveillance for VHL, the extent to which that advice is consistent with published surveillance guidelines, the degree of compliance and factors associated significantly with undercompliance.

\section{Study part I}

On the basis of self-report data, we found that all participants clinically and/or molecular genetically diagnosed with VHL, and the majority of those at $50 \%$ risk, received advice to undergo periodic surveillance for VHL. The majority reported being fully compliant with the advice given. Reported reasons for not complying with the surveillance advice were fear of the MRI scan, fear that surveillance would uncover a tumor, absence of symptoms, and the personal need for rest between periods of illness and/or hospitalization.

For the majority of respondents, the self-reported advice was only partially consistent with published guidelines. Approximately half were advised to undergo all major surveillance procedures but with a frequency that deviated from the guidelines. For the remaining $50 \%$, one or more of the major surveillance procedures was not advised, with a median of one of five missing examinations per individual (e.g., MRI of the cerebellum).

\section{Study part II}

Radiologic surveillance data from the medical records indicated that a substantial percentage of individuals did not fulfill the criteria for minimal compliance and, therefore, did not undergo surveillance as recommended by the national guidelines: between approximately one quarter and one third for screening for CNS lesions, and approximately $50 \%$ for screening for visceral lesions. Of those not appropriately screened for CNS lesions, the majority underwent too few examinations $(<2)$ in a 5-year period. For the radiologic examinations of the upper abdomen, this was approximately $50 \%$. For the remaining undercompliers, the time interval between two consecutive surveillance procedures was too long.

Undercompliance (i.e., too few examinations or too lengthy time intervals) with radiologic examinations for CNS lesions was associated significantly with (having reported) receiving advice that deviated from the national guidelines and with the hospital responsible for the surveillance. With respect to the latter association, this could reflect hospital budgetary issues (i.e., absence of insurance coverage for preventive surveillance of at-risk individuals), logistical problems (e.g., inability to schedule various screening examinations [of the various disciplines] on the same day and difficulty in scheduling examinations at least 3 months in advance), and individual clinician's personal interpretation of the guidelines (e.g., use of MRI for possible CNS lesions only if the individual is symptomatic). 
Table 6 Variables possibly associated with objective compliance with radiological surveillance methods at the univariate level

\begin{tabular}{|c|c|c|c|c|c|c|}
\hline \multirow{2}{*}{$\begin{array}{l}\text { Variables appropriately } \\
\text { screened }^{a}\end{array}$} & \multicolumn{2}{|c|}{$\begin{array}{l}\text { Ultrasound upper abdomen } \\
\quad(n=64),{ }^{b} N(\%)\end{array}$} & \multicolumn{2}{|c|}{$\begin{array}{l}\text { MRI cerebellum } \\
(n=67), N(\%)\end{array}$} & \multicolumn{2}{|c|}{$\begin{array}{l}\text { MRI myelum } \\
(n=67), N(\%)\end{array}$} \\
\hline & Yes & No & Yes & No & Yes & No \\
\hline $\mathrm{Age}^{c}$ & $38.8(12.5)$ & $39.0(14.0)$ & $38.4(13.6)$ & $39.0(13.5)$ & $39.2(13.5)$ & $37.4(13.5)$ \\
\hline \multicolumn{7}{|l|}{ Gender } \\
\hline Male & $17(53)$ & $15(47)$ & $25(76)$ & $8(24)$ & $23(70)$ & $10(30)$ \\
\hline female & $16(50)$ & $16(50)$ & $26(77)$ & $8(24)$ & $21(62)$ & $13(38)$ \\
\hline \multicolumn{7}{|l|}{ Actual risk } \\
\hline Carrier & $31(55)$ & $25(45)$ & $46(79)$ & $12(21)$ & $39(67)$ & $19(33)$ \\
\hline $50 \%$ at risk & $2(25)$ & $6(75)$ & $5(56)$ & $4(44)$ & $5(56)$ & $4(44)$ \\
\hline \multicolumn{7}{|l|}{ Disease status } \\
\hline Affected & $31(56)^{d}$ & $24(44)^{d}$ & $45(78)$ & $13(22)$ & $39(67)$ & $19(33)$ \\
\hline Unaffected & $2(22)^{d}$ & $7(78)^{d}$ & $6(67)$ & $3(33)$ & $5(56)$ & $4(44)$ \\
\hline \multicolumn{7}{|l|}{ Attending hospital } \\
\hline A & $9(50)$ & $9(50)$ & $16(89)^{e}$ & $2(11)^{e}$ & $16(89)^{e}$ & $2(11)^{e}$ \\
\hline $\mathrm{B}$ & $10(71)$ & $4(29)$ & $14(100)^{e}$ & $0(0)^{e}$ & $14(100)^{e}$ & $0(0)^{e}$ \\
\hline $\mathrm{C}$ & $5(31)$ & $11(69)$ & $9(47)^{e}$ & $10(53)^{e}$ & $2(11)^{e}$ & $17(89)^{e}$ \\
\hline Other & $9(56)$ & $7(44)$ & $12(75)^{e}$ & $4(25)^{e}$ & $12(75)^{e}$ & $4(25)^{e}$ \\
\hline \multicolumn{7}{|l|}{$\begin{array}{l}\text { Self-report advice surveillance } \\
\text { method }\end{array}$} \\
\hline Conform guidelines & $23(52)$ & $21(48)$ & $50(87)^{e}$ & $7(12)^{e}$ & $44(77)^{e}$ & $13(23)^{e}$ \\
\hline Deviating from guidelines & $10(50)$ & $10(50)$ & $1(10)^{e}$ & $9(90)^{e}$ & $0(0)^{e}$ & $10(100)^{e}$ \\
\hline Perceived benefits ${ }^{c}$ & $18.5(2.9)$ & $18.9(3.6)$ & $18.9(3.0)$ & $18.2(3.8)$ & $19.0(3.0)$ & $18.2(3.6)$ \\
\hline Perceived barriers ${ }^{c}$ & $14.2(3.5)$ & $13.9(3.6)$ & $14.0(3.7)$ & $14.8(3.6)$ & $14.3(3.7)$ & $13.9(3.6)$ \\
\hline \multicolumn{7}{|l|}{$\begin{array}{l}\text { Perceived risk or developing } \\
\quad \text { a tumor }{ }^{f}\end{array}$} \\
\hline Low & $5(50)$ & $5(50)$ & $8(73)$ & $3(27)$ & $7(64)$ & $4(36)$ \\
\hline Moderate & $14(48)$ & $15(52)$ & $22(73)$ & $8(27)$ & $20(67)$ & $10(33)$ \\
\hline High & $14(58)$ & $10(42)$ & $20(80)$ & $5(20)$ & $17(68)$ & $8(32)$ \\
\hline VHL-specific distress (IES) ${ }^{c}$ & $10.0(11.1)^{d}$ & $5.8(6.9)^{d}$ & $7.5(9.0)$ & $10.2(10.1)$ & $7.1(9.3)$ & $10.0(9.3)$ \\
\hline VHL-related worries $(\mathrm{CWS})^{c}$ & $16.4(6.1)^{d}$ & $14.0(4.1)^{d}$ & $15.1(5.1)$ & $16.3(6.1)$ & $15.1(5.4)$ & $16.0(5.2)$ \\
\hline
\end{tabular}

${ }^{a}$ Appropriately screened $=$ screened in concordance with the national guidelines according to the decision rules based on the guidelines.

${ }^{b}$ Or other radiological examination of the upper abdomen.

${ }^{c}$ For these variables, means (SDs) are given.

${ }^{d}$ Borderline significant difference $P \leq 0.10$.

${ }^{e}$ Significant difference $P \leq 0.05$.

${ }^{f}$ One case missing in analysis.

CWS, Cancer Worry Scale.

Failure to comply with surveillance recommendations (screening delay) for VHL is a cause of concern. As mentioned previously, CNS lesions and RCC are the main causes of morbidity and mortality in patients with VHL. ${ }^{3-7}$ In the case of $\mathrm{RCC}$, to reduce the risk of metastasis, treatment is advised for tumors with a maximum diameter of $3 \mathrm{~cm}^{2}$. CNS lesions are, in general, only treated if symptomatic, with surgical resection as standard treatment.2,27 There is no clear evidence that the patient truly benefits from screening for CNS lesions. However, it has been reported that with periodic surveillance for CNS lesions, it is possible to detect changes in tumors, based on tumor size and growth rate, to predict future symptoms. ${ }^{28}$ This provides the possibility for excision at an earlier stage, before severe neurologic deficits occur. ${ }^{28}$ This is of potential value, because, in general, it is observed that preoperative symptoms are irreversible. ${ }^{11}$ Additionally, one study has suggested that screening delay can increase the risk of interval tumors. ${ }^{12}$ For example, it has been reported that a biannual time interval 
between two consecutive radiologic CNS examinations results in an average risk of $7 \%$ of developing an interval tumor. ${ }^{12}$ It is likely that longer intervals will result in increased risk.

\section{Study limitations}

Several limitations of this study should be noted. First, the sample size was relatively small. However, we attempted to capture the entire VHL population (from families with a pathogenic VHL mutation) in the Netherlands and achieved a high response rate $(77 \%)$. Second, much of the data generated in this study were based on self-report. However, several recent studies of medical screening behavior have reported generally high levels of agreement between self-report data and medical chart audits. $^{29-32}$ Third, the cross-sectional nature of the study only allows us to speak of statistical associations, rather than causal attributions. Fourth, for practical reasons, we were only able to assess objective compliance for the radiologic examinations, and not for the ophthalmologic examination, biochemical blood tests, and 24-hour urine analysis. However, we included three surveillance methods for which the data were most reliable and clinically relevant. Fifth, a minority of the high-risk VHL family members may have had periodic surveillance in a local, nonacademic hospital in which we were unable to collect data. It is unclear as to whether inclusion of data from these latter hospitals would affect the compliance estimates reported in this study and, if so, in which direction. Finally, the choice of cutoff for defining objective noncompliance can always be the subject of debate.

\section{Clinical relevance}

Our results indicate that the majority of individuals diagnosed with, or at high risk of, VHL report having received advice to undergo regular surveillance. The majority indicate being fully compliant with that advice. However, for the majority of individuals, the advice that they reported having received was not entirely consistent with published guidelines. Additionally, based on medical record data, delay in screening was observed for a substantial number of individuals.

To summarize, our results suggest that patients are not noncompliant with surveillance guidelines due to high levels of worry or distress or because they do not see benefits in undergoing screening. Rather, the variability in screening behavior seems to be related primarily to variability in the advice given for periodic surveillance and the various hospitals where patients are being screened. If we aspire to screen patients appropriately, we need to recognize the variability in advice and procedures followed across hospitals, and ultimately address the various barriers (logistical, financial, and attitudinal) to ensure that hospitals comply with contemporary screening guidelines.

Efforts should focus on improving the provision of advice based on available guidelines and on minimizing screening delay. Several concrete steps could be taken in this regard. First, VHL families may benefit from the availability of a "case manager" (e.g., a specially trained nurse practitioner) who could serve as the primary contact for patients with VHL and relatives in the hospital, periodically assess the medical and psychosocial needs of the patients, coordinate multidisciplinary care, and supervise the planning of regular surveillance.

Second, because of the low prevalence and complexity of VHL, it might be better to concentrate on the surveillance and treatment of patients with VHL to a limited number of specialized academic centers in the Netherlands. In the report "Quality of Cancer Care" of the Signaling Committee Cancer of the Dutch Cancer Society, it is stated that "treatment in hospitals of sufficiently large volumes of specific patient populations, de- creases the morbidity and mortality rate." 33 The same may hold for complex rare hereditary cancer syndromes, such as VHL.

Third, if feasible, a "one stop outpatient clinic" for regular surveillance could be introduced. ${ }^{34}$ Additionally, those at $50 \%$ risk need to better understand that the absence of symptoms is not a reason to delay screening. Ongoing research about genophenotype correlations might, in the future, lead to tailored advice for periodic surveillance depending on the VHL mutation. ${ }^{35}$ However, until then, it is important that those individuals diagnosed with or at high risk of VHL undergo periodic surveillance in accordance with existing guidelines. This will facilitate early detection of VHL manifestations and appropriate treatment that can have a salutary effect on both the clinical prognosis and the quality of life of the patient.

\section{ACKNOWLEDGMENTS}

This study was supported by the Dutch Cancer Society (Grant number NKI 2005-3209). The authors are grateful to all respondents who participated in this study. They thank Prof. Dr. C.J.M. Lips for his comments on an earlier draft of this article.

\section{REFERENCES}

1. Hes FJ, Los M, van der Luijt RB. From gene to disease; Von Hippel-Lindau disease. Neth J Med 2002;146:1364-1367.

2. Lonser RR, Glenn GM, Walther M, et al. Von Hippel-Lindau disease. Lancet 2003;361:2059-2067.

3. Maher ER, Yates JR, Harries R, et al. Clinical features and natural history of von Hippel-Lindau disease. $O J$ Med 1990;77:1151-1163.

4. Lamiell JM, Salazar FG, Hsia YE. von Hippel-Lindau disease affecting 43 members of a single kindred. Medicine (Baltimore) 1989;68:1-29.

5. Maddock IR, Moran A, Maher ER, et al. A genetic register for von HippelLindau disease. J Med Genet 1996;33:120-127.

6. Neumann HP, Eggert HR, Scheremet R, et al. Central nervous system lesions in von Hippel-Lindau syndrome. J Neurol Neurosurg Psychiatry 1992;55:898-901.

7. Richard S, Campello C, Taillandier L, Parker F, Resche F. Haemangioblastoma of the central nervous system in von Hippel-Lindau disease. French VHL Study Group. J Intern Med 1998;243:547-553.

8. Latif F, Tory K, Gnarra J, et al. Identification of the von Hippel-Lindau disease tumor suppressor gene. Science 1993;260:1317-1320.

9. Hes FJ, van der Luijt RB, Lips CJ. Clinical management of Von HippelLindau (VHL) disease. Neth J Med 2001;59:225-234.

10. The VHL handbook: what you need to know about VHL, 4th international edition. Boston: VHL Family Alliance, 2005.

11. Glasker S. Central nervous system manifestations in VHL: genetics, pathology and clinical phenotypic features. Fam Cancer 2005;4:37-42.

12. Poulsen ML, Budtz-Jorgensen E, Bisgaard ML. Surveillance in von HippelLindau disease (vHL). Clin Genet 2010;77:49-59.

13. Priesemann M, Davies KM, Perry LA, et al. Benefits of screening in von Hippel-Lindau disease - comparison of morbidity associated with initial tumours in affected parents and children. Horm Res 2006;66:1-5.

14. Wittebol-Post D, Hes FJ, Lips CJ. The eye in von Hippel-Lindau disease. Long-term follow-up of screening and treatment: recommendations. J Intern Med 1998;243:555-561.

15. Rasmussen A, Alonso E, Ochoa A, et al. Uptake of genetic testing and long-term tumor surveillance in von Hippel-Lindau disease. BMC Med Genet 2010;11:4

16. Lammens C, Bleiker E, Verhoef S, et al. Psychosocial impact of Von Hippel-Lindau disease: levels and sources of distress. Clin Genet 2010;77: 483-491.

17. Becker MH. The health belief model and sick role behavior. Health Educ Monogr 1974;2:409-419.

18. Maddux JE, Rogers RW. Protection motivation and self-efficacy: a revised theory of fear appeals and attitude change. J Exp Soc Psychol 1983;19:469-479.

19. Lerman C, Seay J, Balshem A, Audrain J. Interest in genetic testing among first-degree relatives of breast cancer patients. Am J Med Genet 1995;57: 385-392.

20. Champion VL. Instrument development for health belief model constructs. ANS Adv Nurs Sci 1984;6:73-85.

21. Kash KM, Holland JC, Halper MS, Miller DG. Psychological distress and surveillance behaviors of women with a family history of breast cancer. J Natl Cancer Inst 1992;84:24-30.

22. Madalinska JB, van Beurden M, Bleiker EM, et al. Predictors of prophylactic bilateral salpingo-oophorectomy compared with gynecologic screening use in BRCA1/2 mutation carriers. J Clin Oncol 2007;25:301-307. 
23. Brom D, Kleber RJ. De schok verwerkingslijst [Impact of Event Scale]. Ned Tijdschr Psychol 1985;40:164-168.

24. Horowitz MJ. Stress-response syndromes: a review of posttraumatic and adjustment disorders. Hosp Community Psychiatry 1986;37:241-249.

25. Douma KF, Aaronson NK, Vasen HF, et al. Psychological distress and use of psychosocial support in familial adenomatous polyposis. Psychooncology 2010;19:289-298.

26. Lerman C, Kash K, Stefanek M. Younger women at increased risk for breast cancer: perceived risk, psychological well-being, and surveillance behavior. Monogr Natl Cancer Inst 1994;16:171-176.

27. Asthagiri AR, Mehta GU, Zach L, et al. Prospective evaluation of radiosurgery for hemangioblastomas in von Hippel-Lindau disease. Neuro Oncol $2010 ; 12: 80-86$

28. Ammerman JM, Lonser RR, Dambrosia J, Butman JA, Oldfield EH. Long-term natural history of hemangioblastomas in patients with Von Hippel-Lindau disease: implications for treatment. J Neurosurg 2006; $105: 248-255$.
29. Baier M, Calonge N, Cutter G, et al. Validity of self-reported colorectal cancer screening behavior. Cancer Epidemiol Biomarkers Prev 2000;9:229-232.

30. Bleiker EM, Menko FH, Taal BG, et al. Screening behavior of individuals at high risk for colorectal cancer. Gastroenterology 2005;128:280-287.

31. Douma KF, Bleiker EM, Aaronson NK, et al. Long-term compliance with endoscopic surveillance advice for familial adenomatous polyposis (FAP). Colorectal Dis 2010;12:1198-1207.

32. Madlensky L, McLaughlin J, Goel V. A comparison of self-reported colorectal cancer screening with medical records. Cancer Epidemiol Biomarkers Prev 2003;12:656-659.

33. Signalling Committee Cancer. Quality of oncology care in the Netherlands. Amsterdam: Dutch Cancer Society, 2010

34. Fraser L, Watts S, Cargill A, Sutton S, Hodgson S. Study comparing two types of screening provision for people with von Hippel-Lindau disease. Fam Cancer 2007;6:103-111.

35. Friedrich CA. Von Hippel-Lindau syndrome. A pleomorphic condition. Cancer 1999;86:2478-2482. 